\title{
Observations of fast anisotropic ion heating, ion cooling, and ion recycling in large-amplitude drift waves
}

\author{
S. J. Sanders, P. M. Bellan, and R. A. Stern a) \\ California Institute of Technology, Mail Code 128-95, Pasadena, California 91125
}

(Received 12 August 1997; accepted 26 November 1997)

\begin{abstract}
Large-amplitude drift wave fluctuations are observed to cause severe ion temperature oscillations in plasmas of the Caltech Encore tokamak [J. M. McChesney, P. M. Bellan, and R. A. Stern, Phys. Fluids B 3, 3370 (1991)]. Experimental investigations of the complete ion dynamical behavior in these waves are presented. The wave electric field excites stochastic ion orbits in the plane normal $(\perp)$ to $\mathbf{B}$, resulting in rapid $\perp$ heating. Ion-ion collisions impart energy along (\|) $\mathbf{B}$, relaxing the $\perp-\|$ temperature anisotropy. Hot ions with large orbit radii escape confinement, reaching the chamber wall and cooling the distribution. Cold ions from the plasma edge convect back into the plasma (i.e., recycle), causing further cooling and significantly replenishing the density depleted by orbit losses. The ion-ion collision period $\tau_{i i} \sim T^{3 / 2} / n$ fluctuates strongly with the drift wave phase, due to intense $(\approx 50 \%)$ fluctuations in $n$ and $T$. Evidence for particle recycling is given by observations of bimodal ion velocity distributions near the plasma edge, indicating the presence of cold ions $(0.4 \mathrm{eV})$ superposed atop the hot $(4-8 \mathrm{eV})$ plasma background. These appear periodically, synchronous with the drift wave phase at which ion fluid flow from the wall toward the plasma center peaks. Evidence is presented that such a periodic heat/loss/recycle/cool process is expected in plasmas with strong stochastic heating. () 1998 American Institute of Physics.
\end{abstract}

[S1070-664X(98)01603-6]

\section{INTRODUCTION}

In recent years, stochastic heating of plasmas has been extensively studied, both theoretically ${ }^{1-3}$ and experimentally, ${ }^{4-8}$ because of its relevance to nonlinear dynamics in general and to anomalous plasma transport in particular. Almost without exception, experimental work on stochastic heating has been performed in carefully controlled "test" plasmas, i.e., collisionless, well-confined plasmas where the effects of particle Hamiltonian chaos could be examined without interference from collisional transport processes or other complicating effects. The few experiments which have been conducted in "nonideal" plasmas examined only single-particle chaos, and not its effect on global properties of the underlying plasma. ${ }^{6,9}$ Similarly, the large majority of theoretical work in the literature (Refs. 1-3) considers collisionless, well-confined plasmas, although one recent numerical paper addresses both stochasticity and ion-neutral collisions. ${ }^{10}$

Many plasmas of interest, however, are neither collisionless, homogeneous, nor quiescent. For example, Ono et al. found that both stochasticity and collisions were active in tokamak ion heating by radially injected, high-frequency Bernstein waves. $^{11}$ Nonlocal (inhomogeneous) effects such as ion orbit losses and other edge physics have recognized importance in fusion plasmas. ${ }^{12}$ In spite of the fundamental importance of these transport processesHamiltonian stochasticity, collisions, and nonlocal effectsthere have been no detailed experimental investigations of

${ }^{a)}$ Permanent address: University of Colorado, Boulder, CO 80309. particle kinetics in plasmas where several are simultaneously active.

This paper presents investigations of plasma ion dynamics driven by intense electrostatic drift waves in the Caltech Encore tokamak. We have measured ion kinetic distribution functions $f(\mathbf{x}, \mathbf{v}, t)$ when the drift wave amplitude was far above the threshold for stochastic ion heating. ${ }^{6} \mathrm{We}$ observe that although the single-particle ion motion is chaotic in this regime, bulk ion properties such as density and temperature oscillate strongly and coherently near the plasma edge, where the wave amplitude peaks. We observe further that the phases and amplitudes of these oscillations are not consistent with the ideal adiabatic or isothermal oscillations assumed in standard drift wave theories. Neither can the global plasma behavior be understood from the perspective of singleparticle chaos alone. Rather, an interplay of stochastic heating, collisions, orbit loss, and recycling together appear to explain the oscillations: The drift waves cause rapid stochastic ion heating in two dimensions, and the "stochastic" energy is shared among the three degrees of ion freedom via a highly time-dependent collisional process. Thereafter, the very large stochastic orbits destroy ion confinement, as the hottest ions are rapidly ejected, leaving behind a cooled plasma. Finally, the wave electric field periodically convects significant numbers of cold particles from the plasma edge back into the bulk plasma (i.e., "recycles" them), causing additional cooling while replenishing the plasma density depleted by orbit losses. This heat/loss/recycle/cool process repeats periodically, resulting in coherent ion density and temperature fluctuations. The numerous heat transport mechanisms reported here were observed via time- 
and space-resolved laser-induced fluorescence (LIF) spectroscopy. ${ }^{13}$

Section II describes the Encore device as well as the diagnostics and the experimental layout and control system. Section III gives a brief review of drift waves and stochastic heating. Section IV presents the experimental data for cyclic ion heating, cooling, and recycling in the presence of the drift waves. Section V gives additional discussion and concluding remarks.

\section{APPARATUS AND EXPERIMENTAL SETUP}

\section{A. The Encore tokamak}

The experimental work was performed in the Caltech Encore tokamak, which has minor radius $a=12.7 \mathrm{~cm}$, major radius $R=38 \mathrm{~cm}$, relatively low toroidal magnetic field $B_{\varphi}$ $\$ 1200 \mathrm{G}$, and high repetition rate $(15 \mathrm{~Hz})$. The high repetition rate and generous optical access make Encore wellsuited for LIF measurements of ion kinetics. Argon plasmas were used because many convenient LIF schemes are available for Ar II ions.

The toroidal field for these experiments was $B_{\varphi}=300 \mathrm{G}$, giving ion and electron cyclotron frequencies of $\Omega_{i} / 2 \pi$ $=1.2 \times 10^{4} \mathrm{~s}^{-1}$ and $\Omega_{e} / 2 \pi=5 \times 10^{9} \mathrm{~s}^{-1}$. Other relevant parameters were plasma current $I_{p}=1-1.5 \mathrm{kA}$, density $n=1$ $-5 \times 10^{12} \mathrm{~cm}^{-3}$ fully ionized Ar II, and ion and electron temperatures $T_{i}=1-10 \mathrm{eV}$ and $T_{e}=5-15 \mathrm{eV}$. The toroidal magnetic field was generated in steady state by a $50 \mathrm{~kW}$ dc power supply and the Ohmic heating $(\mathrm{OH})$ current was generated by an audio frequency amplifier $(15 \mathrm{~kW} \mathrm{rms})$.

In addition to LIF, Langmuir probes were used extensively for this work. The plasma was sufficiently cool to allow full probe insertion without causing probe damage. Probes were available at an array of toroidal locations and could be positioned within a poloidal cross section by computer-controlled stepping motors. Measurement of the probe current-voltage characteristic allowed determination of $n, T_{e}$, plasma potential $\phi_{p}$, and floating potential $\phi_{f}$. Unmagnetized probe theory could be used because the probe was typically collecting ions, and the ion gyroradius was much larger than both the ion Debye length and probe radius. ${ }^{14}$

Plasma parameters for a typical discharge are plotted in Fig. 1. Figure 1(a) shows the computer-generated voltage wave form amplified and applied across the primary windings of the $\mathrm{OH}$ transformer. Figure 1(b) shows the induced toroidal loop voltage $V_{\text {loop }}$ and Fig. 1(c) the resulting plasma current $I_{p}$ for a typical shot. Figure 1(d) displays the ion saturation current collected by a Langmuir probe inserted near the plasma edge. The fluctuations $\left(\omega / 2 \pi=4 \times 10^{3} \mathrm{~s}^{-1}\right)$ evident in the saturation current of Fig. 1(d) result from the presence of large-amplitude drift-Alfvén waves, as shown by Fredrickson and Bellan. ${ }^{15}$ The drift waves will be discussed extensively beginning in Sec. III A.

\section{B. Laser induced fluorescence in encore}

LIF was used to measure ion distribution functions $f(\mathbf{x}, \mathbf{v}, t)$ in the presence of the drift waves. The LIF laser
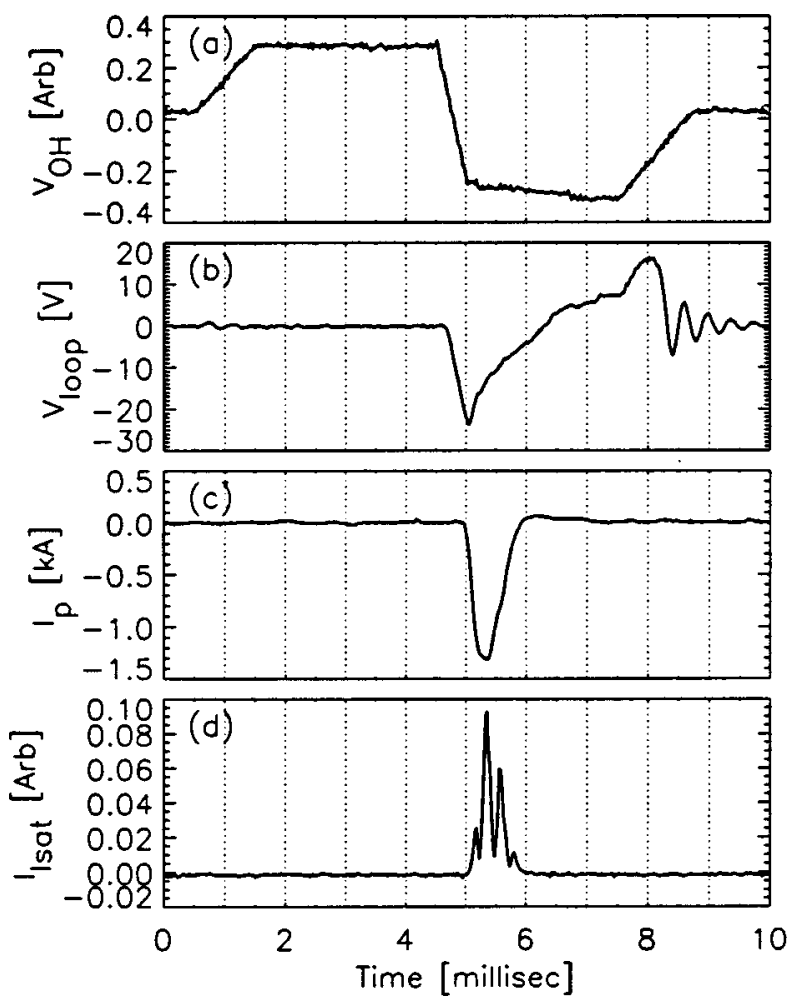

FIG. 1. Typical shot parameters: (a) Voltage wave form applied to $\mathrm{OH}$ transformer. (b) Toroidal loop voltage. (c) Plasma current, measured by Rogowski coil. (d) Ion saturation current to Langmuir probe.

system consisted of a narrow-band, tunable dye laser (Lambda Physik FL2001E) pumped by a frequency-doubled ND:Yag (Laser Photonics MY-32). The spectra of Ar II ions in Encore was primarily Doppler broadened. Pressure, Stark, and Zeeman broadening were negligible compared to the Doppler width at all but the lowest observed ion temperatures. Thus the absorption spectrum was a map of the ion velocity distribution through the Doppler shift formula $\lambda_{\text {lab }}$ $=\lambda * \times\left(1-v_{k} / c\right)$. Here $\lambda *$ is the resonant absorption wavelength of ions at rest, $\lambda_{\text {lab }}$ is the ion resonance as seen in the lab frame, $c$ is the speed of light, and $v_{k}$ is the ion velocity (in the lab frame) along the laser beam direction $\hat{k}$. Scanning the laser across the ion Doppler spectrum on successive plasma shots yields the $v_{k}$-dependence of $f(\mathbf{x}, \mathbf{v}, t)$ at fixed $\mathbf{x}$ and $t$. The $10 \mathrm{~ns}$ laser pulse width gave excellent time resolution compared to the drift wave period $\tau_{\text {wave }} \approx 230 \mu \mathrm{s}$, and the laser trigger timing could be indexed with $50 \mathrm{~ns}$ resolution to determine the time evolution of $f$.

Two LIF schemes were employed for this work. These are shown schematically in Fig. 2. Each scheme allowed measurement of the ion velocity distribution of one Ar II metastable state $\left(3 d^{2} G_{9 / 2}\right.$ and $3 d^{4} F_{9 / 2}$, respectively; these differ in energy by $1.7 \mathrm{eV}$ ). The measured velocity distributions for ions in these two states were found to agree well, suggesting that the LIF data is representative of the bulk ion velocity distribution.

The measurement of ion dynamics synchronous with the drift wave phase was complicated by substantial shot-to-shot jitter in the drift wave phase. Because the LIF diagnostic required many shots to obtain a complete measurement of $f$ 
(a)

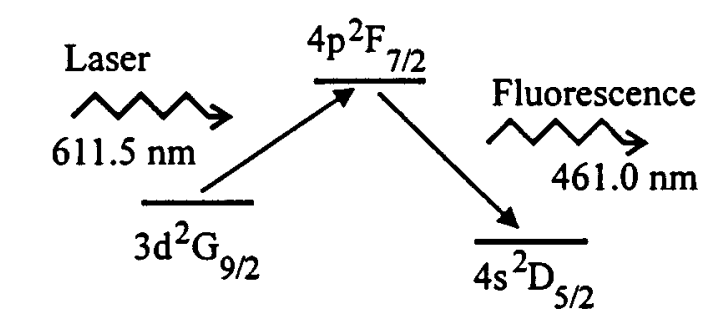

(b)

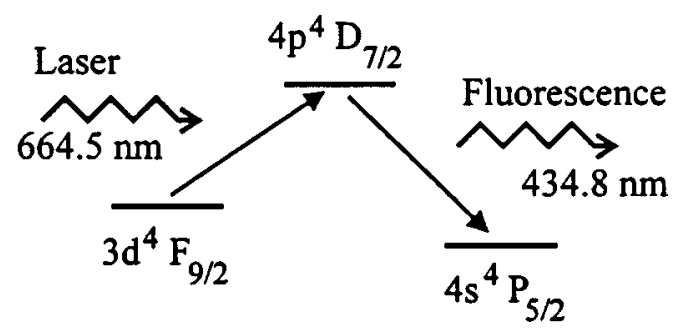

FIG. 2. Two Ar II LIF schemes used for this work.

at fixed $\mathbf{x}$ and $t$, it was crucial to maintain a fixed laser trigger relative to the drift wave phase for thousands of consecutive shots. This synchronization could not be achieved by triggering the laser with a phase reference (such as a Langmuir probe) because the shot-to-shot wave phase jitter was larger than the adjustment range of the pump laser timing triggers. To solve this problem, we developed a novel data acquisition system which monitored in real time the wave phase relative to the laser trigger $t_{\mathrm{YAG}} \cdot{ }^{16}$ This system identified discharges with waves having excessive phase delay relative to $t_{\mathrm{YAG}}$ and rejected LIF data from those shots. Conversely, discharges having phase delay within a predefined tolerance were accepted, and data from those shots were used to deduce $f(\mathbf{v}, t)$. Shots could be screened for anomalously high or low amplitude as well. This method therefore ensured that the waves used for averaging purposes were selected from a subset of discharges having low phase and amplitude scatter.

\section{Experimental setup}

Figure 3 shows the experimental arrangement used to measure multidimensional ion distribution functions. The dye laser beam was split, and the two resulting beams (e.g., beams 1 and 2, shown in Fig. 3) were directed across the same plasma volume at right angles to each other so as to probe two orthogonal velocity components of $f$. Beam 2 (cf. Fig. 3) was delivered to the plasma through a long optical fiber which introduced a $300 \mathrm{~ns}$ time delay relative to beam 1. This delay was much smaller than the drift wave period but allowed the fluorescence signals due to beams 1 and 2 to be analyzed independently by separately gated boxcar integrators.

Spatial resolution was $1 \mathrm{~cm}^{3}$, as determined by the intersection of the viewing optics field and the laser beam, and thus was much smaller than the $20 \mathrm{~cm}$ poloidal drift wave-

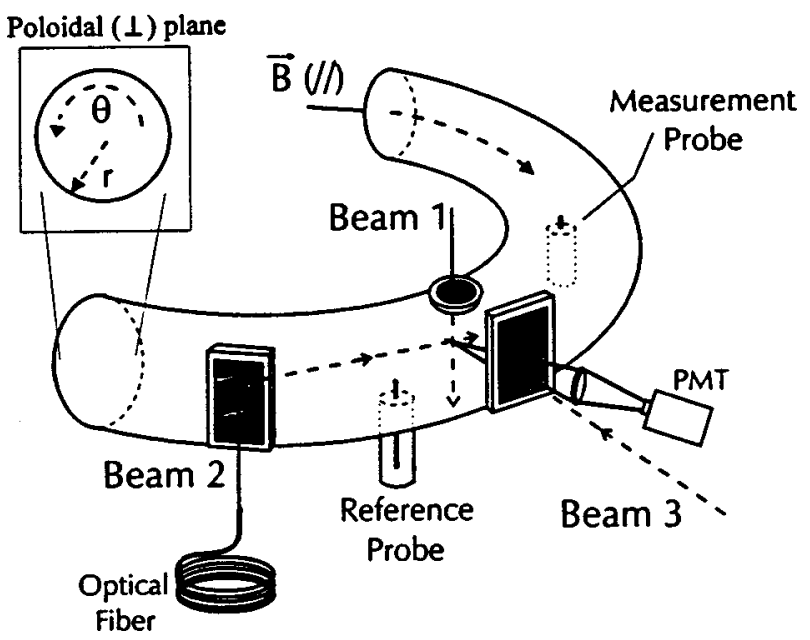

FIG. 3. Schematic of experimental setup. The toroidal magnetic field direction is labeled $\|$. The poloidal and minor-radial directions, respectively, labeled $\perp r$ and $\perp \theta$ in the text, are shown in the inset. The "Reference", Langmuir probe provided phase reference (cf. Sec. III), and the "Measurement" probe determined density and plasma potential.

length. The spectral bandwidth of the pulsed dye laser permitted resolution of ion temperatures at least as low as 0.3 $\mathrm{eV}$.

\section{DRIFT WAVES}

\section{A. Introduction}

Drift waves are normal modes of inhomogeneous magnetized plasmas. Drift waves grow at the expense of free energy stored in plasma pressure and temperature gradients. ${ }^{17}$ Since all magnetically confined plasmas are necessarily finite in extent, drift waves are ubiquitous in laboratory plasma devices and have been dubbed "the universal instability." 18

Derivations of the drift wave dispersion relation are given by a number of authors. ${ }^{15,19}$ The real part of the wave frequency is given by

$$
\omega_{r}=\frac{\omega_{e *}}{1+b} .
$$

Here $\omega_{e *}=k_{\perp} \kappa_{B} T_{e} \partial n / \partial r\left(m_{e} \Omega_{e} n\right)^{-1}$ is the electron diamagnetic drift frequency, $b \equiv k_{\perp}^{2} \kappa_{B} T_{e}\left(m_{i} \Omega_{i}^{2}\right)^{-1}, m_{i}$ and $m_{e}$ are the ion and electron masses, $\kappa_{B}$ is Boltzmann's constant, and $k_{\perp}$ is the wave vector perpendicular to the magnetic field. A correction to Eq. (1) due to the magnetic character of the wave has been neglected, since for Encore parameters this correction is of order $1 \%$.

The drift wave growth rate $\omega_{i}$ results from axial electron drift, ${ }^{20}$ Landau damping, ${ }^{21}$ and electron-ion collisions. ${ }^{15}$ In Encore, the electron-drift term dominates, so that $\omega_{i}$ has the form

$$
\omega_{i}=\frac{\omega_{*}^{2}}{k_{\|} v_{T e} \omega_{r}} \frac{\pi^{1 / 2} k_{\varphi} u_{\varphi e}}{(1+b)^{3}},
$$

where $u_{\varphi e}$ is the electron toroidal drift velocity, $k_{\|}$is the wave vector along $\mathbf{B}$, and $v_{T e}=\left(\kappa_{B} T_{e} / m_{e}\right)^{1 / 2}$ is the electron thermal speed. The electron drift is related to the plasma current 

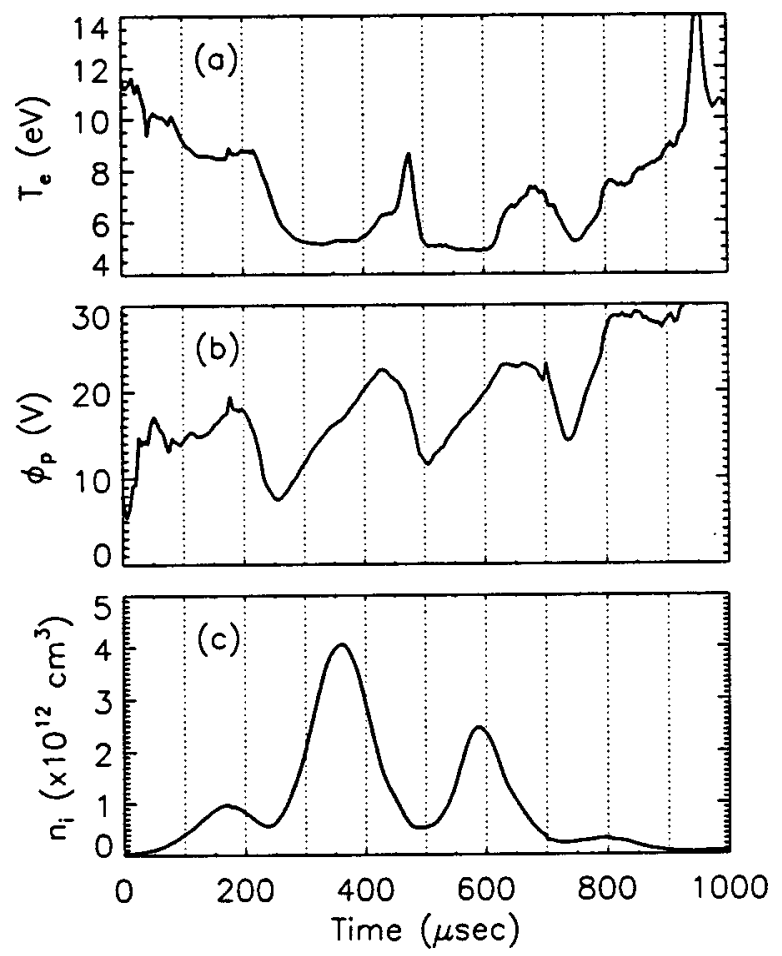

FIG. 4. Measured evolution of (a) electron temperature, (b) plasma potential, and (c) ion density in the presence of large-amplitude drift waves. Parameters were measured by Langmuir probe $4 \mathrm{~cm}$ from the plasma edge.

through $u_{\varphi e}=I_{p} / n q$, meaning that the wave amplitude $\tilde{\phi}$ can be controlled by varying the $\mathrm{OH}$ voltage which drives $I_{p}$. Large-amplitude, coherent waves can be excited for $I_{p}$ $\gtrsim 0.3 \mathrm{kA}$.

The drift wave spatial structure has the form

$$
\phi(r, \theta, z, t)=\widetilde{\phi} f(r) e^{i(m \theta+n \varphi-\omega t)},
$$

where $(r, \theta, \varphi)$ are the minor radial, poloidal, and toroidal directions, respectively (cf. Fig. 3). The poloidal mode number is $m=2$, and the toroidal mode number is $n=1$ (see Ref. 15 for plots of spatial structure), giving wave vector components $k_{\theta}=2 / r_{0}=30 \mathrm{~m}^{-1}$ and $k_{\varphi}=1 / R=2.6 \mathrm{~m}^{-1}$, where $r_{0}$ is the radius of maximum wave amplitude. Thus $k_{\theta} \gg k_{\varphi}$, and the wave propagates nearly normal to $B_{\varphi}$.

Typical fluctuations in electron temperature $T_{e}$, plasma potential $\phi$, and ion density $n$ are shown in Fig. 4. These were measured by a Langmuir probe located near the plasma edge during a typical shot with large-amplitude drift wave activity. The amplitude of the potential fluctuation is $\widetilde{\phi}=6$ $\mathrm{V}$. Thus the wave electric field, $\widetilde{\mathbf{E}}=-\nabla \widetilde{\phi}$, has components $\widetilde{E_{\theta}} \approx 1.8 \mathrm{~V} / \mathrm{cm}$ and $\widetilde{E_{\varphi}} \approx 0.18 \mathrm{~V} / \mathrm{cm}$.

\section{B. Ion stochasticity}

It is well known that large-amplitude electrostatic plasma waves can lead to stochastic particle orbits and heating. Some of the first theoretical treatments were given by Smith and Kaufman, ${ }^{1}$ Karney and Bers, ${ }^{2}$ and Drake and Lee. ${ }^{3}$ The first experimental investigation of stochastic heating was reported by Doveil, who observed stochastic electron heating by standing waves. ${ }^{4}$ Stochastic ion heating has been observed in the presence of driven ion Bernstein waves, ${ }^{5}$ and driven ion cyclotron waves. ${ }^{7,8}$ McChesney, Stern, and Bellan used LIF to measure ion distribution functions in the presence of the drift waves in Encore. ${ }^{6}$ They found that for large $\widetilde{\phi}$, Encore ions were rapidly and stochastically heated by the drift waves.

Reference 6 considered a slab model for ion dynamics in the presence of the drift waves. In rectangular coordinates, i.e., $(r, \theta) \rightarrow(x, y)$ and $\varphi \rightarrow z, \mathbf{B}=B_{0} \hat{z}$ and $\mathbf{E}=-\nabla \phi$, with $\phi(x, y, z, t)=\phi_{0} \cos \left(k_{y} y-\omega t\right)$. Here the parallel wave number $k_{z}$ has been neglected, since in Encore, $k_{y} \gg k_{z}$. The Lorentz equations of motion for a single ion are

$$
\dot{v}_{x}=\Omega_{i} v_{y}, \quad \dot{v}_{y}=-\Omega_{i} v_{x}+\frac{q \widetilde{\phi}}{m_{i}} k_{y} \sin \left(k_{y} y-\omega t\right) .
$$

The first equation may be integrated and the integration constants eliminated by redefining the $y$ and $t$ origins. It is useful to write the resulting equations in dimensionless form, by normalizing times to the cyclotron period and distances to the wavelength. Defining $\tau \equiv \Omega_{i} t, \chi \equiv k_{y} x$, and $\xi \equiv k_{y} y$, we have

$$
\begin{aligned}
& \frac{d \chi}{d \tau}=+\xi \\
& \frac{d^{2} \xi}{d \tau^{2}}=-\xi+\alpha \sin (\xi-\nu \tau),
\end{aligned}
$$

where $\alpha \equiv q \phi_{0} k_{y}^{2} / m_{i} \Omega_{i}^{2}$ and $\nu \equiv \omega / \Omega_{i}$.

Ion orbits become stochastic for wave amplitudes above the threshold $\alpha \gtrsim 1$. Physically, the chaotic motion is due to particles being temporarily trapped in a potential well of the drift wave and subsequently de-trapped by the pull of the magnetic field. The onset of stochasticity for large $\alpha$ can be seen explicitly by solving Eqs. (2) and (3) numerically and making Poincaré section plots of the trajectories. Figure 5 shows representative phase trajectories, in un-normalized units, for a range of perturbation amplitudes $\alpha$. It is clear from Figs. 5(c) and 5(f) that for $\alpha=0.9$, stochastic orbits cover a wide range of ion velocity space. Note that this entire region of velocity space is accessible to ions in the bulk of the distribution. The width of the stochastic region gives the maximum velocities attainable by these bulk ions. By construction of Poincare plots for various $\alpha$, one finds this maximum bulk velocity to be ${ }^{22}$

$$
v_{\max } \approx \frac{\Omega_{i}}{k_{y}}(\alpha+1.9) \text {. }
$$

This holds for field strengths above the stochastic threshold, i.e., when $\alpha \geqslant 1$. Finally, it is also clear that the motion is stochastic along both $v_{x}$ and $v_{y}$, although the wave is polarized along $\hat{y}$. In contrast, motion along $z$ is regular (even when $k_{z}$ is finite), provided only one electrostatic mode propagates along $\mathbf{B}^{23,24}$ Thus stochastic heating is expected to be highly anisotropic, heating only in the directions normal to $\mathbf{B}$.

Using laser-induced fluorescence we have measured ion distribution functions along all three velocity dimensions 


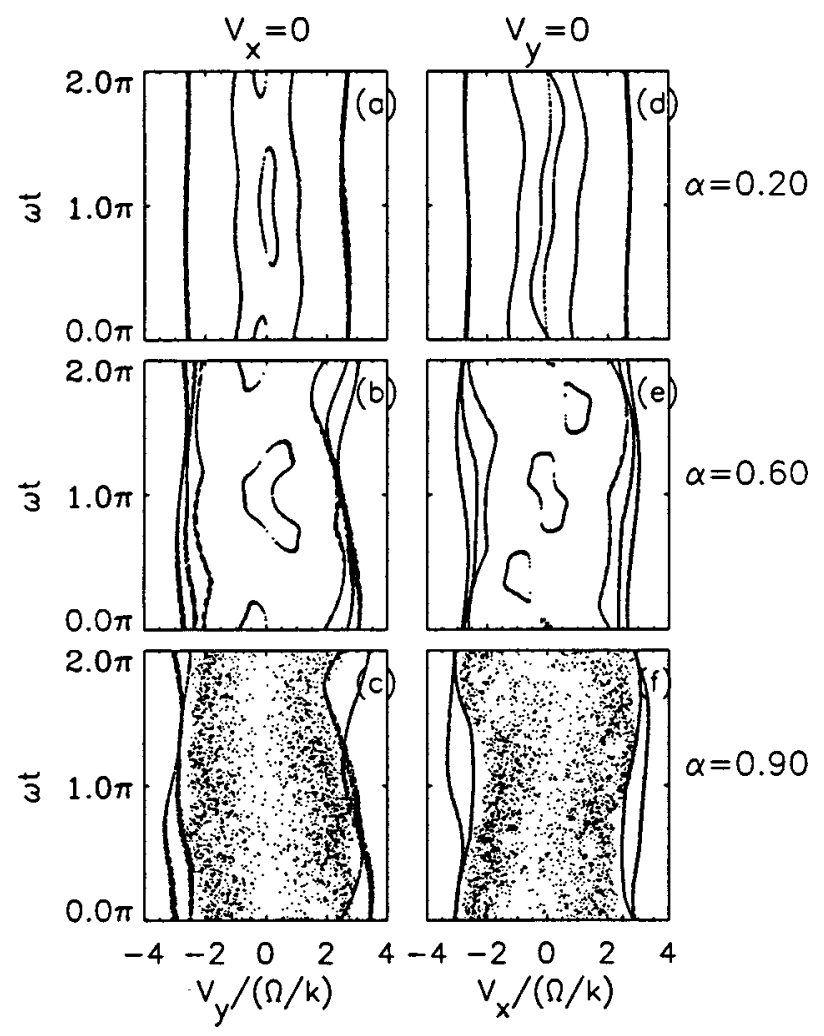

FIG. 5. Poincare sections for ion orbits in the drift wave fields. The phase planes are $[(\mathrm{a}),(\mathrm{b}),(\mathrm{c})] v_{x}=0$ and $[(\mathrm{d}),(\mathrm{e}),(\mathrm{f})] v_{y}=0$. Each curve corresponds to a unique ion initial condition. Plots are generated by 4 ions making roughly 5000 punctures.

with high space and wave phase resolution. Measurements were performed for wave amplitudes $\alpha \gtrsim 2$. These measurements enabled detailed examination of the transfer of energy among the various degrees of ion freedom and several new aspects of the large-amplitude drift wave heating process have been observed for the first time. The experimental observations are presented in the next section.

\section{OBSERVATION OF FAST ION HEATING CYCLES}

\section{A. Ion heating perpendicular $(\perp)$ to $\mathbf{B}$}

Figure 6(a) plots the measured time evolution of two components of the perpendicular ion temperature $T_{\perp r}$ and $T_{\perp \theta}$, in the presence of large-amplitude drift waves. ( $T$ with no species subscript is understood to refer to ions.) Ion density $n(t)$ measured simultaneously by a Langmuir probe is shown in Fig. 6(b) for comparison. These measurements were performed $4 \mathrm{~cm}$ from the tokamak wall, where the drift wave amplitude was largest. Here the space potential fluctuation was $\widetilde{\phi}_{p}=6$ volt, giving $\alpha=2.3$, well above the stochastic heating threshold. Very strong ion temperature fluctuations, coherent with the drift wave, are evident in the figure. There is a large phase delay $\left(\approx 130^{\circ}\right)$ between the $n$ and $T$ fluctuations, and we find that this phase relationship is inconsistent with what would follow from an adiabatic ion equation of state, namely $T n^{1-\gamma}=$ const, where $\gamma$ is the ratio of ion specific heat at constant pressure to that at constant volume. For instance, a two-dimensional adiabatic oscilla-
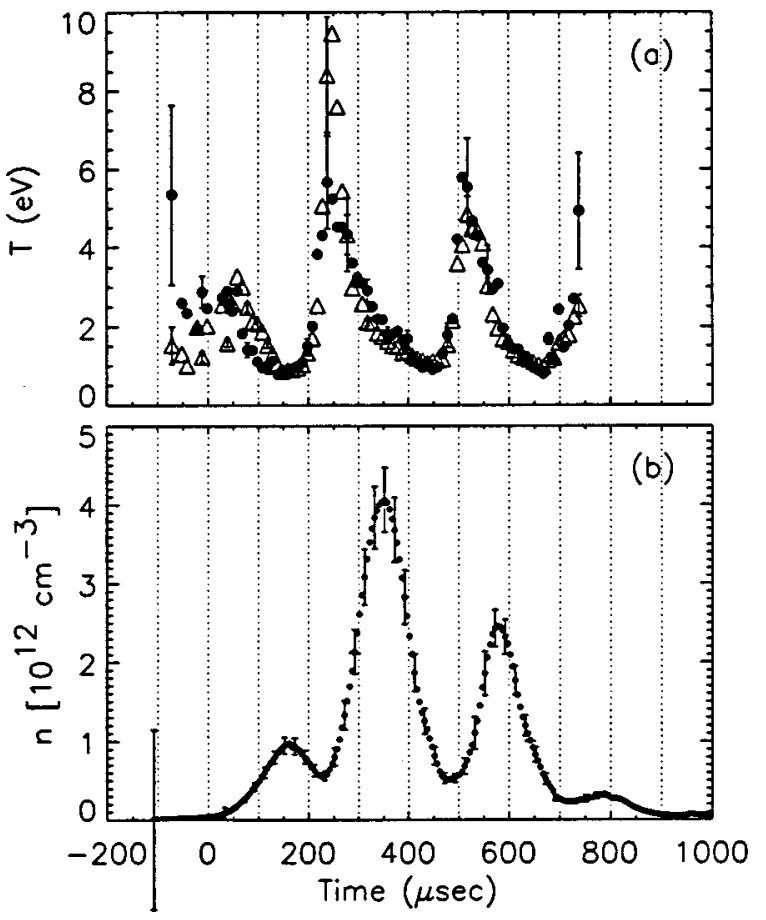

FIG. 6. $T_{\perp r}(t), T_{\perp \theta}(t), n(t)$ measured $4 \mathrm{~cm}$ from plasma edge. (a) LIFmeasured ion temperatures: $T_{\perp \theta}(\triangle), T_{\perp r}(\bullet)$. (b) Ion density measured by Langmuir probe. The probe was located $18^{\circ}$ toroidally downstream from the LIF location, and probe data were adjusted to account for the wave phase delay in traversing this distance.

tion would have $\gamma=2$, and the ideal adiabatic state equation would yield $T_{\perp} / n=$ const, where $T_{\perp}=\left(T_{\perp r}+T_{\perp \theta}\right) / 2$. The experimental data of Figs. 6(a) and 6(b), however, indicate the opposite phase relation: $T_{\perp}$ increases whilst $n$ decreases. Thus the ion temperature fluctuations are evidently nonadiabatic and cannot be explained by standard theories which close the chain of dynamical equations by an ideal equation of state. In Secs. IV B and IV D we present direct experimental evidence that both heat conduction by collisions and external heat sinks (the chamber wall) are instrumental in determining the ion temperature. Hence the ideal state equations, which are valid only when such effects are negligible, are not expected to apply in this case. Note, however, that at all wave phases, $T_{\perp r}(t)$ and $T_{\perp \theta}(t)$ are equal, within experimental error. The isotropic nature of the heating within the $\perp$ plane is consistent with stochastic heating, as described in Sec. III B.

The extremely fast heating rate observed here, $\dot{T}_{\perp} \approx 0.1$ $\mathrm{eV} / \mu \mathrm{s}$, is also characteristic of stochastic dynamics. This heating is much faster than can be explained by classical mechanisms, such as equilibration with the hot electron background or direct acceleration by the toroidal electric field. Electrons are rapidly heated $(\approx 5 \mathrm{eV} / \mu \mathrm{s})$ by the Ohmic heating field and therefore seemingly offer a rich supply of energy for ions. However the collisional exchange of energy between electrons and ions is slow. The ion-electron energy exchange time is given by ${ }^{25} \tau_{i e}^{E}=3.1 \times 10^{8} \mu T_{e}^{3 / 2} / n \Lambda_{i e}$, with $\mu$ the ion mass in amu and $\Lambda_{i e}$ the Coulomb logarithm. Evaluating $\tau_{i e}^{E}$ for typical Encore parameters $\left(T_{e} \sim 7 \mathrm{eV}, n\right.$ $\sim 2 \times 10^{12} \mathrm{~cm}^{-3}$ ) we find $\tau_{i e} \geq 2.1 \mathrm{msec}$, or about 10 times 


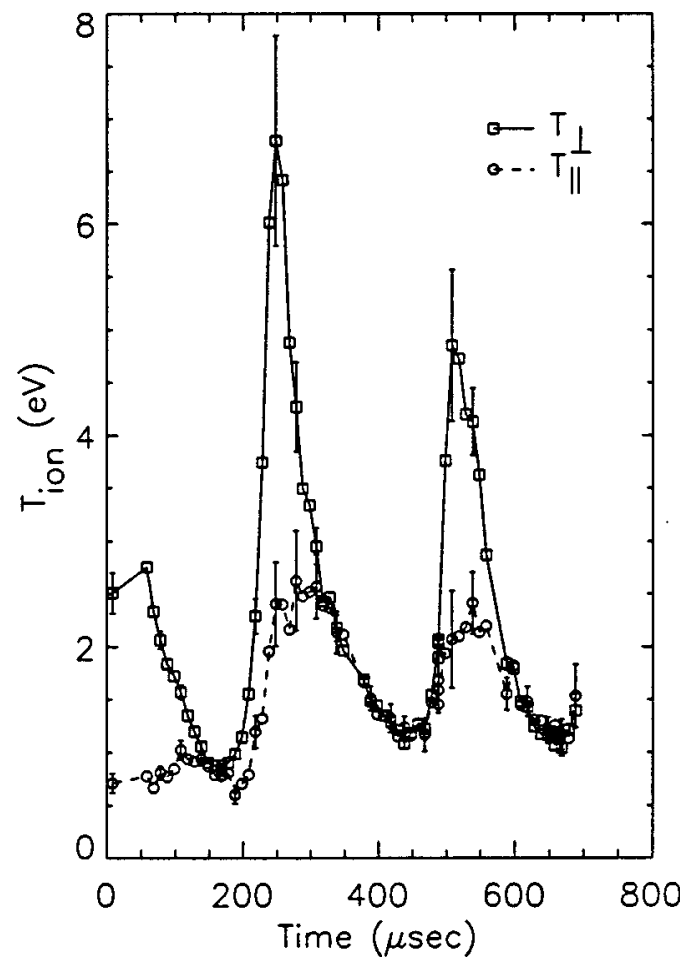

FIG. 7. Measured ion temperatures: $T_{\perp}(\square)$ and $T_{\|}(\bigcirc)$.

the wave period. The ion heating rate due to collisions with hot electrons is then $\dot{T}=\left(T_{e}-T\right) / \tau_{i e}^{E} \lessgtr 0.003 \mathrm{eV} / \mu \mathrm{s}$, accounting for only $3 \%$ of the observed ion heating.

Direct ion acceleration by the $\mathrm{OH}$ electric field, followed by randomizing collisions with electrons, could also give ion heating. In a uniform electric field $E$, ions acquire a drift velocity $u_{d}=q E \tau_{e i} / m_{i}$. Collisions with electrons then thermalize the energy at the rate $\dot{T}_{i} \approx m_{i} u_{d}^{2} / 2 \tau_{e i}=q^{2} E^{2} \tau_{e i} / 2 m_{i}$. For the $\mathrm{OH}$ toroidal field in Encore, $E_{\|} \sim V_{\text {loop }} / 2 \pi R \approx 8$ $\mathrm{V} \mathrm{m}^{-1}$, this gives only $\dot{T}_{i} \leqslant 10^{-4} \mathrm{eV} / \mu \mathrm{s}$, a negligible heating rate. Thus the observed perpendicular ion heating cannot be explained by classical mechanisms. Rather, the observed heating rate is consistent with that expected for a stochastic mechanism and with previous measurements of stochastic heating rates. ${ }^{20}$

\section{B. Parallel ion heating}

Figure 7 shows measurements of $T_{\|}$, the ion temperature along the magnetic field. For comparison, $T_{\perp}=\left(T_{\perp r}\right.$ $\left.+T_{\perp \theta}\right) / 2$ is also plotted, using the same $T_{\perp}$ data as in Fig. 6(a). $T_{\|}$also heats rapidly, at a peak rate of $\dot{T}_{\|} \approx 0.035 \mathrm{eV} / \mu \mathrm{s}$. While this rate is a factor of 3 lower than $\dot{T}_{\perp}$, it is still at least 10 times greater than can be explained by the classical mechanisms discussed above. Furthermore, direct heating by the wave electric field should be confined to the $\perp$ plane, because the wave is polarized with $k_{\perp} / k_{\|} \gg 1$ and also because ion dynamics are not stochastic along $\mathbf{B}$.

We now consider parallel heating due to ion-ion collisions relaxing the ion $\perp-\|$ temperature anisotropy. The expectation that collisions are indeed the primary parallel heating mechanism is supported by two prominent features seen in Fig. 7: (i) The $T_{\perp}-T_{\|}$anisotropy is most pronounced in the first $100 \mu$ s of the discharge. This anisotropy is consistent with the low density and hence low ion-ion collision rate at these early times. For $n \leqslant 5 \times 10^{11} \mathrm{~cm}^{-3}$ the ion-ion collision time is $\tau_{i i}>100 \mu \mathrm{s}$, and little collisional energy transfer from $T_{\perp} \rightarrow T_{\|}$can occur. Note that the large $T_{\perp}$ indicates that stochastic heating is strong at these early times, and it also contributes to the low collision rate, since $\tau_{i i}$ $\sim T^{3 / 2}$. (ii) Although the parallel heating is coherent with the perpendicular heating, there is a significant phase delay between the two. This delay contrasts sharply with the case of $T_{\perp r}$ and $T_{\perp \theta}$, which heat simultaneously. Hence the wave is not heating $T_{\|}$directly. Instead, wave energy is transferred to $T_{\|}$after a delay suggestive of a collisional process.

We now give a quantitative model for the evolution of $T_{\|}$. It is assumed that the parallel ion heating is described by

$$
\dot{T}_{\|}(t)=\left(\dot{T}_{\|}\right)_{C}+\left(\dot{T}_{\|}\right)_{L},
$$

where the term labeled $C$ represents collisional equipartition of energy between the three degrees of ion freedom, and the term labeled $L$ represents heat loss due to transport of hot ions out of the plasma. The loss term is included to be consistent with the observation that the ions cool periodically (cf. Fig. 7). This parallel cooling cannot be explained by mere exchange of energy among the three degrees of ion freedom because, as the figure reveals, $T_{\perp}$ and $T_{\|}$decrease simultaneously. Section IV C presents evidence that the cooling is caused by loss of confinement of hot ions with large cyclotron radii. For the moment, we simply note that the loss term must be small compared to the collisional term during the heating phase of the wave, i.e., when $\dot{T}_{\|}>0$; if it were not, then $T_{\|}$could not rise so rapidly during the heating cycle. Thus we neglect the loss term and assume that the parallel heating is attributable to collisions only

$$
\dot{T}_{\|}(t) \simeq\left(\dot{T}_{\|}\right)_{C} .
$$

This equation is understood to hold only when $\dot{T}_{\|}>0$.

Collisions in a fully ionized plasma are described by Fokker-Planck (FP) theory, ${ }^{26}$ which assumes that the cumulative effect of numerous glancing collisions governs particle transport. Note that the use of unmagnetized transport theory is justified when the ratio of ion cyclotron radius to Debye length is large ${ }^{27}$ in Encore, $r_{i} / \lambda_{D i} \approx 4000$ and the unmagnetized theory may be applied. For the unmagnetized case, the rate at which ion-ion collisions relax ion temperature anisotropies was first predicted by Kogan as ${ }^{28}$

$$
\left(\dot{T}_{\|}\right)_{C}=\frac{T_{\perp}-T_{\|}}{\tau_{i i}^{E}(t)},
$$

where

$\tau_{i i}^{E} \equiv \frac{4 m_{i}^{1 / 2} \varepsilon_{0}^{2}\left(\pi \kappa_{B} T_{\|}\right)^{3 / 2}}{e^{4} n \Lambda_{i i}}\left[\frac{A^{2}}{-3+A^{-1 / 2}(A+3) \tan ^{-1}\left(A^{1 / 2}\right)}\right]$.

Here $\varepsilon_{0}$ is the permittivity of free space and $A=T_{\perp} / T_{\|}-1$ is a measure of the ion temperature anisotropy, assumed to be positive. For $A<0$, the term $A^{-1 / 2} \tan ^{-1}\left(A^{1 / 2}\right)$ must be re- 


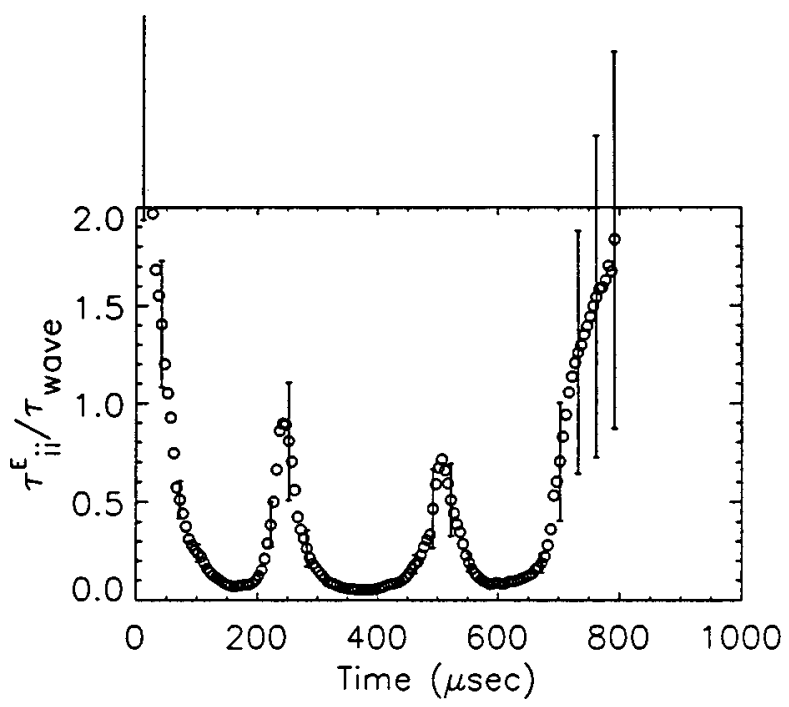

FIG. 8. Ion-ion collision period $\tau_{i i}^{E}$, according to Eq. (9), normalized to the wave period $\tau_{\text {wave }} \approx 230 \mu \mathrm{s}$.

placed by $(-A)^{-1 / 2} \tanh ^{-1}(-A)^{1 / 2}$. In the limit $A \rightarrow 0$, the term in brackets evaluates to $15 / 4$, and the energy exchange period reduces to $4 / 5$ of the classical Spitzer period. ${ }^{29}$

Anisotropic temperature relaxations (ATR) have been observed in pure electron plasmas by Hyatt, Driscoll, and Malmberg, ${ }^{30}$ and also by Beck, Fajans, and Malmberg. ${ }^{31}$ Anderegg et al. have observed ATR in a pure magnesium ion plasma with $\lambda_{D}>r_{L} \cdot{ }^{32}$ All of those experiments were conducted in quiescent, Penning-trapped plasmas with densities below $10^{9} \mathrm{~cm}^{-3}$. ("Quiescent" plasmas refer to plasmas containing little or no wave activity. Fluctuations of less than 5\% were reported in Refs. 30-32.) Many plasmas of interest, however, are not quiescent, and the equipartitioning of anisotropic particle energy in plasmas with significant wave activity has been scarcely examined. In the Bernstein-wave heating experiments in the JIPPT-II-U tokamak, ${ }^{11}$ both parallel and perpendicular temperatures were measured, but the time evolution of the anisotropy was not analyzed in detail. We now compare the Fokker-Planck predictions with Encore heating data, obtained when the drift wave amplitude was far above the threshold for stochastic heating.

It is instructive to begin by plotting $\tau_{i i}^{E}$, calculated from Eq. (8) using the measured $n, T_{\perp}$, and $T_{\|}$. Figure 8 displays $\tau_{i i}^{E}$ normalized to the drift wave period $\tau_{\text {wave }}$. One sees that the collision period fluctuates strongly in time, resulting from the nearly $180^{\circ}$ phase shift between local ion density and temperature (cf. Fig. 6). The amplitude of these fluctuations clearly demonstrates that the wave is strongly perturbing the underlying kinetic properties of the plasma: The wave transforms the plasma from weakly collisional $\left(\tau_{i i}^{E} \approx \tau_{\text {wave }}\right)$ to highly collisional $\left(\tau_{i i}^{E} \ll \tau_{\text {wave }}\right)$ on time scales shorter than a wave period.

Next, Eqs. (7) and (8) are combined to yield a single equation for the evolution of $T_{\|}(t)$. This equation is solved numerically using the measured $T_{\perp}(t)$ and $n(t)$ shown in Fig. 6 as inputs. Figure 9 shows the result of the calculation for three wave heating cycles. The Fokker-Planck prediction for $T_{\|}$is plotted as the solid curve in each heating cycle. The

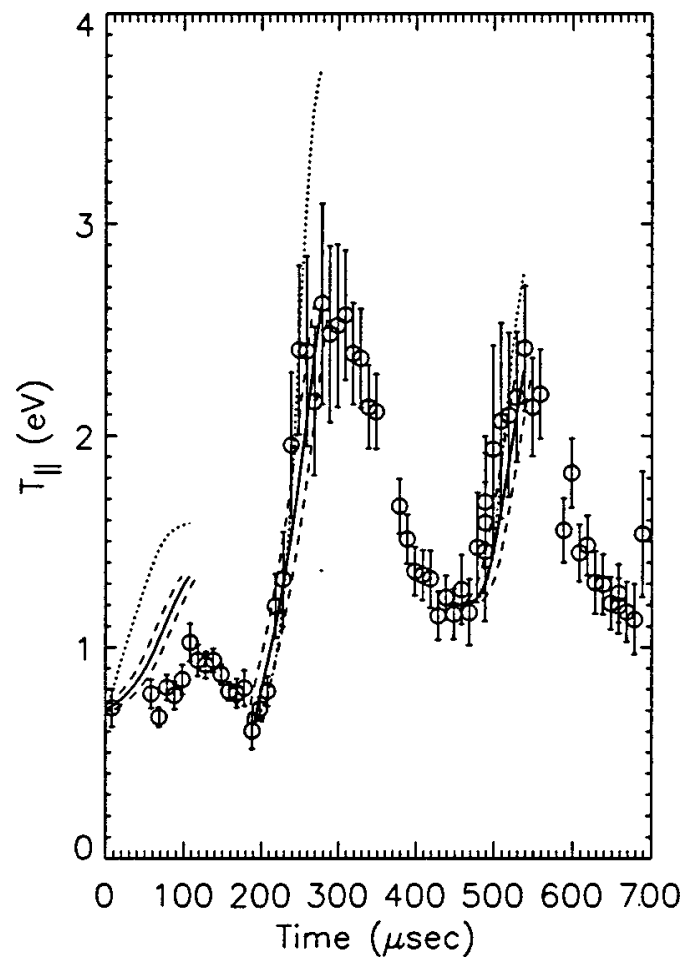

FIG. 9. $T_{\|}$: Fokker-Planck prediction (solid curve), measured data (circles), and prediction using average collision time (dotted curve). Dashed curves show uncertainty in prediction, due primarily to wave phase jitter.

dashed curves indicate the prediction's uncertainty, owing primarily to the shot-to-shot fluctuations in the drift wave phase. The circles are the measured data. Overall, the amplitude and phase of the predicted $T_{\|}(t)$ agree well with the data, ${ }^{33}$ indicating that the dominant mechanism driving the $\perp \rightarrow \|$ energy transport is indeed Fokker-Planck-type collisions. For comparison, Fig. 9 also shows a calculation of $T_{\|}(t)$ obtained from the time-averaged value of $\tau_{i i}^{E}$ (dotted curves). Here the agreement is markedly worse, underscoring that the instantaneous character of $\tau_{i i}^{E}$ must be taken into account. Fokker-Planck theory has not been extensively tested in the presence of strong waves, so the apparent consistency between the theory and our results in this dynamic regime is noteworthy.

\section{Ion cooling}

A glance at Fig. 7 reveals that the ions cool periodically. For example, in the time range $t=250 \rightarrow 400 \mu \mathrm{s}$, ions lose energy at a rate $\dot{T} \simeq-0.035 \mathrm{eV} / \mu \mathrm{s}$. This cooling cannot be due to ions returning energy to the drift wave, since stochastic heating is irreversible. ${ }^{3}$ Nor can collisions with electrons explain the cooling rate: as noted in Sec. IV B, the exchange of energy between ions and electrons is a factor of 10 too slow, and moreover, the electrons are relatively hot. Collisions with cold neutrals can cool ions, but the density of neutrals, assuming only $90 \%$ ionization, is at least two orders of magnitude too low to explain the observed cooling rate. Finally, radiation cannot directly sap ion kinetic energy.

Transport of energetic ions out of the plasma can produce ion cooling. Ions which reach the cold chamber wall are 
expected to recombine and eventually recycle as cold atoms. ${ }^{34,35}$ Loss and recycling would cause simultaneous cooling along all three velocity dimensions, in accord with our observations: The close agreement between $T_{\perp}$ and $T_{\|}$at the phases of minimum temperature suggests that the ions periodically reach an equilibrium with a common cold reservoir. Thereafter, $T_{\perp}$ and $T_{\|}$heat through different mechanisms and therefore at different rates, as described above. This process can explain how $T_{\perp}$ and $T_{\|}$periodically agree and yet have different bulk heating rates.

To estimate the cooling rate such a loss process would cause, we assume that an ion loses all its kinetic energy upon striking the wall. The ion cooling rate can then be estimated by integrating over trajectories which reach the wall. Such a calculation is difficult for conditions in Encore, where the stochastic orbits cannot be represented analytically. However, lower and upper bounds for the loss rate can be predicted by considering orbits in the two limiting cases below:

(1) The classical limit of non-stochastic, magnetized orbits. For motion in tokamak fields, classical orbits consist of gyromotion together with guiding center drifts due to field curvature, gradient, etc. In this case, the distance an ion can stray from a field line is determined by the relatively weak poloidal $(\theta)$ component of the magnetic field, rather than the strong toroidal component. An upper bound for this distance is $r_{\theta}=m v_{\|} / q B_{\theta},{ }^{36}$ where $v_{\|}$is the ion velocity in the toroidal direction. Thus, ions within a distance $d \leqslant r_{\theta}$ from the wall have reasonable probability of escaping confinement. The velocity condition for escape is then $v_{\|} \gtrsim \Omega_{\theta} d$, where $\Omega_{\theta}=q B_{\theta} / m$. The rate at which these ions carry energy to the wall is obtained by integrating over the velocity distribution $f(\mathbf{v})$

$$
(\dot{T})_{L}^{\text {classical }} \approx \frac{\Omega_{\theta}}{2 \pi} \int_{\left|v_{\|}\right|>\Omega_{\theta} d} \frac{m \mathbf{v}^{2}}{2} f(\mathbf{v}) d^{3} \mathbf{v},
$$

where it is assumed that the time for all ions to escape is roughly the cyclotron period $2 \pi / \Omega_{\theta}$. For $f(\mathbf{v})$ an anisotropic Maxwellian $\left[f \propto \exp \left(-v_{\|}^{2} / T_{\|}^{2}-v_{\perp}^{2} / T_{\perp}^{2}\right)\right]$, the integral evaluates to

$$
\begin{aligned}
(\dot{T})_{L}^{\text {classical }}= & \frac{\Omega_{\theta}}{2 \pi}\left\{T_{\|}\left[\frac{2}{\pi^{1 / 2}} a e^{-a^{2}}+\operatorname{erfc}(a)\right]\right. \\
& \left.+T_{\perp} \operatorname{erfc}(a)\right\},
\end{aligned}
$$

where $a=\Omega_{\theta} d / v_{\|}^{\text {th }}, v_{\|}^{\text {th }}$ is the parallel thermal velocity, and $\operatorname{erfc}()$ is the complementary error function. Equation (9) is an expression for ion power transfer to the wall. For Encore parameters $\left(B_{\theta}=30 \mathrm{G}, d=4 \mathrm{~cm}\right)$, Eq. (9) predicts a peak heat loss rate of $\dot{T} \approx-0.007 \mathrm{eV} / \mu \mathrm{s}$, about a factor of 5 lower than what is observed.

(2) The strongly stochastic limit, in which the stochastic region of velocity space is very wide. In this limit, the ions become very energetic and have concomitantly large gyroradii. Hence we treat ion trajectories as straight-line orbits toward the wall to calculate an absolute upper bound on the cooling rate due to orbit loss. An ion reaches the wall in time
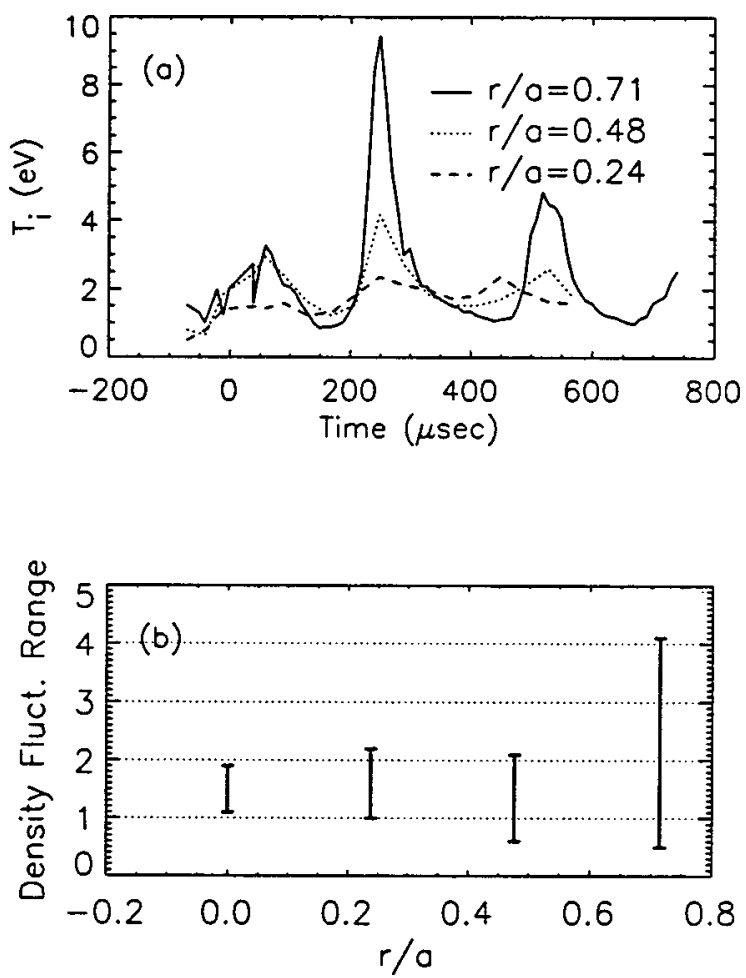

FIG. 10. (a) Variation of ion temperature $T$ with minor radius. Radii are normalized to the machine radius $a=13 \mathrm{~cm}$. Near the machine center $(r / a=0.24), T$ executes small oscillations $(\sim 30 \%)$ about a pedestal of 1.8 $\mathrm{eV}$. Near the edge, $T$ oscillates from above $5 \mathrm{eV}$ to below $1 \mathrm{eV}$ each cycle. (b) Ion density fluctuation range, as a function of minor radius. Bars extend from the "pedestal" density (lower end) to peak instantaneous density (upper end).

$t$ if $v_{\text {wall }} \geqslant d / t$ where $v_{\text {wall }}$ is the velocity toward the wall and again $d$ is the ion's distance from the wall. Assuming all the ions are initially located together and integrating over a Maxwellian predicts a loss rate of

$$
\begin{aligned}
\dot{T}_{L}^{\text {stochastic }}(t) & \approx \frac{d}{d t} \int_{v_{\text {wall }}>d / t} \frac{m \mathbf{v}^{2}}{2} f(\mathbf{v}) d^{3} \mathbf{v} \\
& =m \frac{d^{3}}{v_{\text {wall }}^{\text {th }} t^{4}} \exp \left[-\frac{m}{2 T}\left(\frac{d}{t}\right)^{2}\right] .
\end{aligned}
$$

The peak loss rate given by Eq. (10) is $\dot{T}_{L}^{\text {stochastic }} \approx-0.15 \mathrm{eV} /$ $\mu \mathrm{s}$, a factor of 5 higher than the cooling rate observed. Thus the measured cooling rate lies between the classical and the strongly stochastic limits for heat loss due to ion transport to the wall.

Moreover, measurements show that the cooling is strongest near the chamber wall, as would be expected for an ion loss mechanism. Figure 10(a) plots the measured ion temperatures at several locations in the plasma. One sees that both the heating and cooling are most extreme near the plasma edge, $r / a \rightarrow 1$. For example, at $r / a=0.24$, i.e., near the torus center, $T_{i}$ rises in the first $100 \mu \mathrm{s}$ to a "pedestal" value of about $1.8 \mathrm{eV}$ and executes small oscillations $(\sim 30 \%)$ about this pedestal. However, near the plasma edge, where the heating is clearly stronger, $T_{i}$ oscillates from a peak of nearly $10 \mathrm{eV}$ to below $1 \mathrm{eV}$, significantly colder 

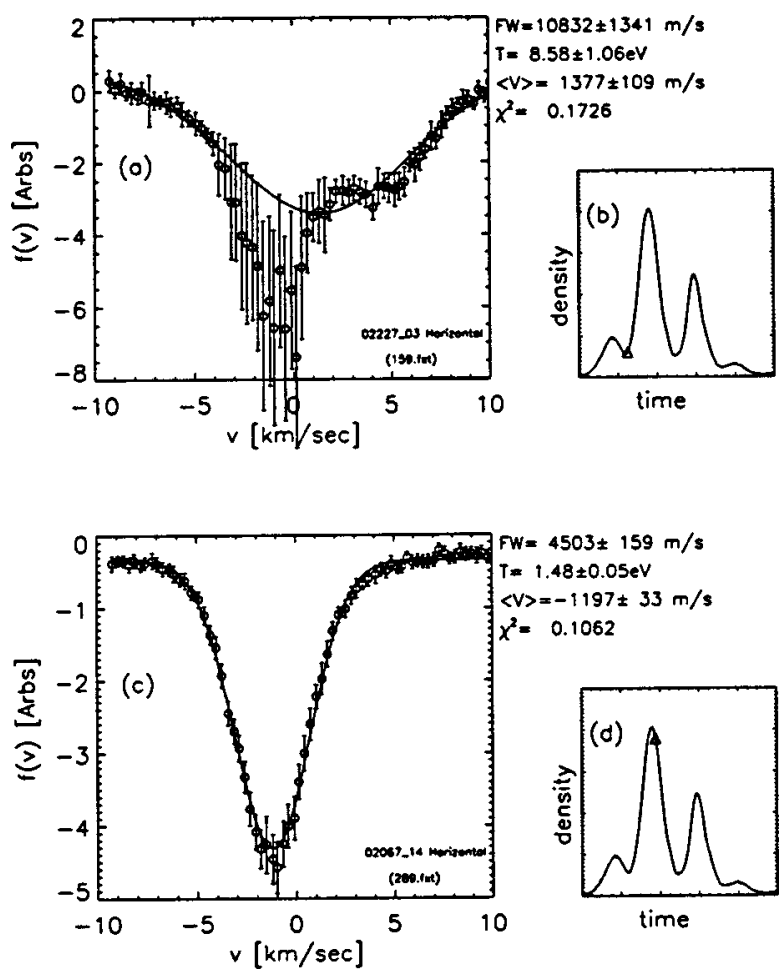

FIG. 11. Shape of poloidal component of velocity distribution, $f\left(v_{\theta}\right)$, near plasma edge when (a) density was local minimum, and (c) density was local maximum. Solid curves show best-fit Maxwellians, and the fitted temperature $T$ and flow velocity $\langle v\rangle$ are given. Measurement times $(\triangle)$ are shown at the right, in (b) and (d), respectively.

than at the plasma center. Ion heating is strongest at the edge because the density gradient, and hence drift wave amplitude, are peaked there; that the cooling is strongest near the edge suggests particle loss. The loss argument is further corroborated by the spatial dependence of the ion density fluctuations. Figure 10(b) compares the amplitude of density fluctuations at several minor radial positions. The data show that at the center the density fluctuations are only $25 \%$ of the equilibrium $\langle n\rangle=1.5 \times 10^{12} \mathrm{~cm}^{-3}$, whereas near the edge the density alternately soars far above $\left(4 \times 10^{12}\right)$ and far below $\left(0.5 \times 10^{12}\right)$ this equilibrium value. Thus, ion cooling by orbit loss is consistent with both the measured cooling rate and the observed spatial dependence of the temperature and density fluctuations.

\section{Ion recycling}

During each wave cycle, significantly non-Maxwellian ion velocity distributions were consistently observed near the chamber wall at wave phases of minimum density. Figure 11(a) shows an example of such a non-Maxwellian distribution. The figure plots $f\left(v_{\theta}\right)$ measured $4 \mathrm{~cm}$ from the chamber wall at a time when the local density was at a minimum; for reference, this sampling time is indicated by the triangle in the density plot of Fig. 11(b). The distribution is clearly nonMaxwellian, with an anomalous concentration of ions near $v_{\theta}=0 .{ }^{37}$ For comparison, Fig. 11(c) shows the same component $f\left(v_{\theta}\right)$ measured at the same spatial location, but sampled when the drift wave density was at a maximum [cf. Fig. 11(d) for sampling time]. The distribution in Fig. 11(c)

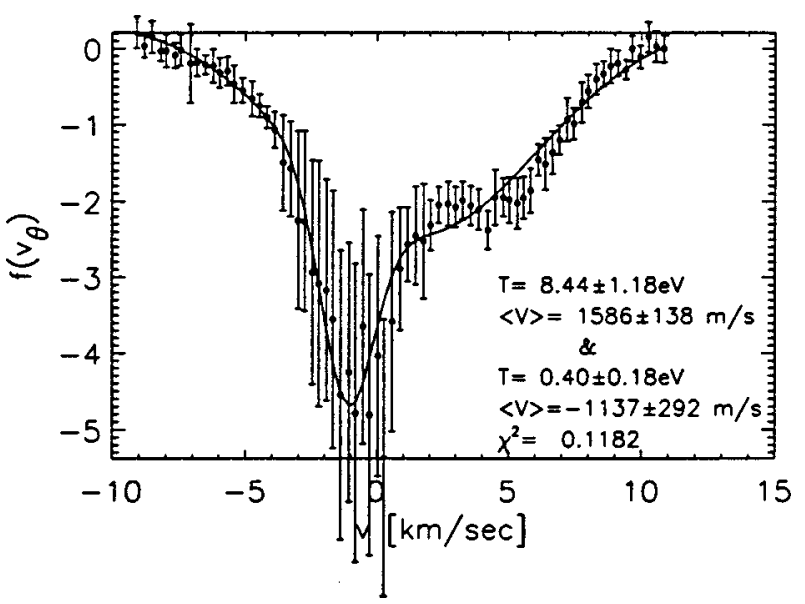

FIG. 12. $\chi^{2}$ fit of $f\left(v_{\theta}\right)$ to sum of two Maxwellians. Fitted temperatures are 8.4 and $0.4 \mathrm{eV}$, respectively.

yields an excellent fit to a Maxwellian, in sharp contrast with the distribution in Fig. 11(a). The experimental procedure and apparatus used to measure these two distributions were identical in every regard except for the time at which the laser was triggered, proving that the observed nonMaxwellian features cannot be artifacts of the measurement technique.

The distribution in Fig. 11(a) is bimodal, and is well fit by a sum of two Maxwellians with independent temperatures, amplitudes, and fluid velocities. The result of a $\chi^{2}$-fit is shown in Fig. 12. The fit is clearly much better than the single-Maxwellian fit shown in Fig. 11(a), and the distribution apparently consists of a group of very cold $(0.4 \mathrm{eV})$ ions superposed atop a very hot $(8.4 \mathrm{eV})$ plasma. The fit also reveals the relative numbers of cold and hot ions. For this distribution, the cold and hot particle densities were found to be roughly equal. Thus the cold ions account for a significant fraction of the total plasma density at $r / a=0.7$.

Such bimodal ion distributions were found only within about 4 to $5 \mathrm{~cm}$ of the chamber wall and only at the drift wave phase of minimum ion density. This is illustrated in Fig. 13(a), which shows how the ratio of cold to hot particle density depends on the drift wave phase. Figure 13(b) shows the total ion density $n_{\text {total }}(t)$, measured at the same location. It is clear that anomalously cold ions appear regularly at the wave phase of minimum density. Low ion density implies long ion-ion collision times (cf. Sec. IV B), and therefore at these wave phases one expects relatively slow relaxation of any non-Maxwellian or bimodal features in $f(\mathbf{v})$. This argument implies that locally bimodal distribution functions can exist at these wave phases, but does not explain their origin.

Insight regarding this origin is given in Fig. 13(c), which plots the time dependence of $u_{r}=\int v_{r} f\left(v_{r}\right) d v_{r}$, the ion fluid velocity along the minor radial direction (experimentally obtained from the Doppler shift in the ion LIF spectrum). These measurements were performed $4 \mathrm{~cm}$ from the chamber wall, with the laser oriented such that $u_{r}<0$ indicates ion flow directed inward from the nearby wall, through the diagnosed volume, and toward the plasma center. As can be seen, the phases having significant ion flux directed inward from the 


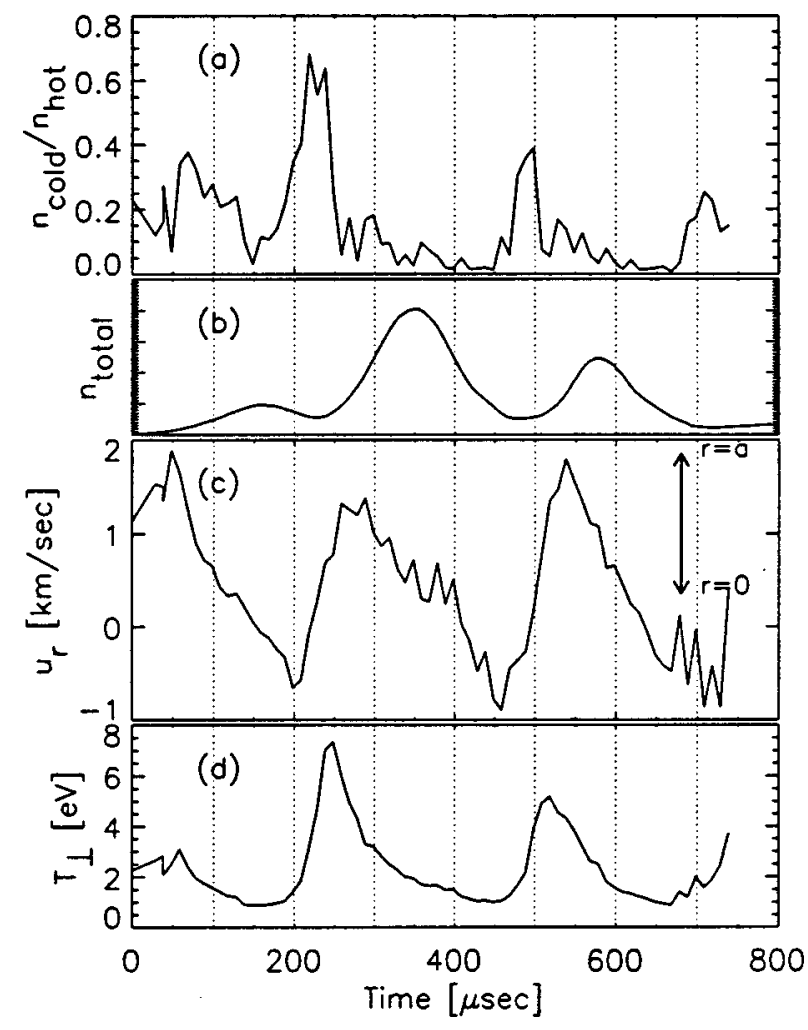

FIG. 13. (a) Measured fractional density of anomalous cold ions. (b) Total ion density. (c) Ion fluid velocity $u_{r}$ along minor radius. Positive velocities represent flow directed toward plasma edge $(r=a)$. (d) Ion temperature. Density and temperature data were given in Fig. 6, and are reproduced here for phase comparison only.

wall are followed closely ( $\Delta t \approx 30-50 \mu \mathrm{s}$ ) by the appearance of cold ions. The observed delay time, $\Delta t \approx 40 \mu \mathrm{s}$, is roughly consistent with the time required for an ion to traverse $4 \mathrm{~cm}$ at typical measured fluid velocities $u_{r} \approx-1$ $\mathrm{km} / \mathrm{s}$. Bailey et al. showed that fluid motion in the Encore drift waves had the same flow pattern as predicted by $\mathbf{E} \times \mathbf{B}$ drift theory but that the measured fluid speed was about an order of magnitude lower than expected, the discrepancy being attributed to finite Larmour radius effects. ${ }^{9}$ The measured $u_{r}$ here is consistent with a similarly reduced $\mathbf{E} \times \mathbf{B}$ flow.

Ions returning from the edge after interaction with the wall, i.e., recycled ions, should be cold relative to the bulk plasma. This is expected because the plasma-wall interaction consists predominantly of cooling processes. For example, ions may recombine or undergo charge-exchange collisions at the wall; they may adsorb to or be absorbed by the wall; and they may reflect or sputter impurities and wall material. ${ }^{38}$ These processes have been studied for noble gases as well. For the case of argon, it is well-known that $\mathrm{Ar}$ readily forms monolayers on a number of surface materials, including graphite, ${ }^{39}$ platinum, ${ }^{40}$ rubidium, ${ }^{41}$ potassium, ${ }^{42}$ and cesium. ${ }^{42}$ Argon ions may also be trapped in the first few layers of surface atoms, as has been observed in silicon ${ }^{43}$ and tungsten ${ }^{44}$ surfaces, for example. The trapping energies for noble gases $(\mathrm{Ne}, \mathrm{Ar}, \mathrm{Kr})$ in tungsten are in the range $2-5 \mathrm{eV},{ }^{44}$ and these trapped atoms may be desorbed by subsequent ion impacts on the wall. ${ }^{45}$ Neon atoms recycling from a carbon limiter by this process have been reported in
Toroidal Experiment for Technically Oriented Research (TEXTOR), with observed Ne velocity distributions indicating a superposition of cold, ion-desorbed atoms $(0.3 \mathrm{eV})$ and hot reflected ones $(15 \mathrm{eV}) .{ }^{34}$ Hence it seems likely that there is an inventory of argon stuck to the Encore chamber wall, and that these cold Ar atoms may be released into the plasma by hot impinging particles.

Argon recycling at a beryllium limiter has been modeled by Brooks. ${ }^{35} \mathrm{He}$ found that impinging $7 \mathrm{eV}$ argon ions recycle primarily by adsorption, followed by thermal desorption at $0.17 \mathrm{eV}$. Although the plasma conditions and surface materials in Refs. 34 and 35 were somewhat different from those in Encore, the similarity between the recycled ion energies found in those references and in the present measurements is noteworthy. We conclude that the temperature of the inward-convecting ions is consistent with the energies expected for recycled particles.

These observations strongly suggest that the drift wave periodically convects cold recycled ions back into the central plasma. This convection peaks when the local plasma density is a minimum, the plasma having been depleted by orbit losses due to stochastic particle acceleration, and the escaping hot ions having released cold argon from the wall. Since the recycled ions comprise a large fraction $(\sim 50 \%)$ of the total ion density, recycling appears to be a significant mechanism for plasma replenishing. ${ }^{46}$

Equilibration between the hot local plasma and the cold recycled ions would be expected to generate some local ion cooling. To check this expectation, the time evolution of the measured ion temperature $T$ is plotted in Fig. 13(d). Comparison of $T(t)$ with the time dependence of the cold ion densities, [cf. Fig. 13(a)], suggests that the onset of ion cooling correlates with the arrival of the recycled ions: Note, for example, the decrease in $T$ starting at $t_{\text {cool }} \simeq 250 \mu$ s following the prominent peak in the cold ion density at $t_{\mathrm{ci}} \simeq 230 \mu \mathrm{s}$. The delay time $t_{\mathrm{cool}}-t_{\mathrm{ci}} \simeq 20 \mu \mathrm{s}$ is roughly comparable to the time required for a cold beam to equilibrate with the background plasma. This occurs by a two-step process: The beam isotropizes on a fast time scale due to transverse diffusion in velocity space and equilibrates with the background plasma on a slower timescale. The transverse diffusion time is given by Trubnikov ${ }^{47}$ and in the limit of a slow beam $\left(m v_{\text {beam }}^{2} / 2\right.$ $\ll T_{\text {plasma }}$ ) may be written approximately as

$$
\tau_{i i}^{\perp}=7.1 \times 10^{6} \frac{\mu^{1 / 2} T_{\mathrm{bkg}}^{1 / 2} \epsilon}{n \Lambda_{i i}}
$$

where $\mu$ is the ion mass in proton units, $\epsilon=m v_{\text {beam }}^{2} / 2$ is the directed beam energy, $T_{\mathrm{bkg}}$ is the background plasma temperature, and energies are in $\mathrm{eV}$. Assuming roughly equal beam and background plasma densities [cf. Fig. 13(a)], we estimate, at the time of peak cold ion flux $\left(T_{\mathrm{bkg}}=1.5 \mathrm{eV}\right.$, $\left.n_{\text {bkg }}=0.4 \times 10^{12} \mathrm{~cm}^{-3}, v_{\text {beam }}=0.8 \mathrm{~km} / \mathrm{s}, \Lambda_{i i} \approx 7\right), \tau_{i i}^{\perp} \approx 3$ $\mu \mathrm{s}$. The temperature equilibration between the resulting cold Maxwellianized beam and the hot background plasma occurs on a timescale ${ }^{48}$

$$
\tau_{i i}^{T}=2.5 \times 10^{6} \frac{\mu^{1 / 2}}{n \Lambda_{i i}}\left(T_{\text {cold }}+T_{\mathrm{bkg}}\right)^{3 / 2} \approx 14 \mu \mathrm{s} .
$$



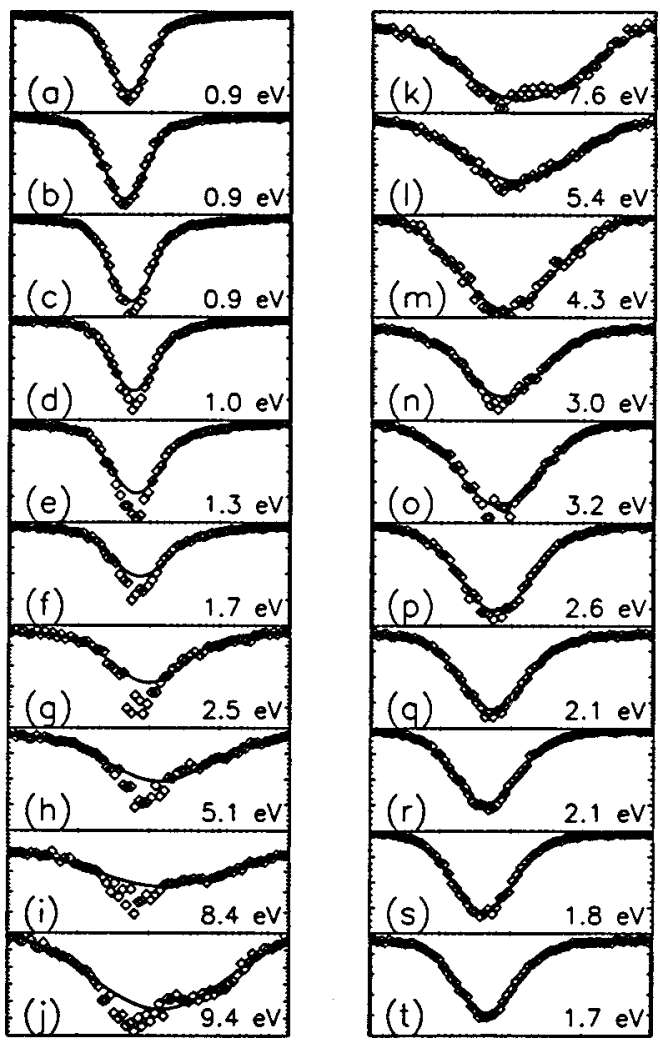

FIG. 14. Time evolution of bimodal ion distribution function. Time interval is $10 \mu$ s per frame, advancing first down the left column (a)-(j), then down the right column $(\mathrm{k})-(\mathrm{t})$. Discrete points are data, and solid curves are bestfit single Maxwellians. The dashed vertical lines indicate $v=0$.

Hence $\tau \approx \tau_{i i}^{\perp}+\tau_{i i}^{T} \approx 17 \mu \mathrm{s}$ is the predicted beam-plasma equilibration time.

The equilibration between the hot and cold ion components can be seen in the time evolution of the distribution function. Figure 14 shows the evolution of $f\left(v_{\theta}, t\right)$ over a $200 \mu$ s time interval centered on a density minimum. Each frame is a snapshot of $f\left(v_{\theta}, t\right)$ at fixed time $t$. The frames are $10 \mu \mathrm{s}$ apart, and the instantaneous temperature inferred from the best-fit (single) Maxwellian is given in the lower right corner of each frame. For reference, the wave phases corresponding to these sampling times are shown by the discrete diamonds on the fluid velocity plot of Fig. 15. As can be seen, the distribution begins to show significant cold ion densities in frame (d), when the fluid velocity directed inward from the wall is $u_{r} \approx-400 \mathrm{~m} / \mathrm{s}$; this time corresponds to the arrival of the first cold ions. The cold ion population builds over the next 30-40 $\mu \mathrm{s}$, as the inward fluid velocity peaks around $u_{r} \approx-800 \mathrm{~m} / \mathrm{s}$ and begins to reverse. The cold prominence on $f\left(v_{\theta}\right)$ then remains visible for the next several frames, relaxing finally by about frame $(\mathrm{m})$. The nonMaxwellian features visible in frames (n) and (o) are clearly much more subtle than those in, e.g., frame (g) and are probably insignificant in light of experimental uncertainties. One other interesting feature of Fig. 15 is that the background temperature continues to rise in spite of the arrival of cold ions: In frames (e)-(j) the temperature rises from 1.3 to 9.4 $\mathrm{eV}$ although the presence of cold recycled ions is clear. This again reflects the low ion collision rate, which is in fact

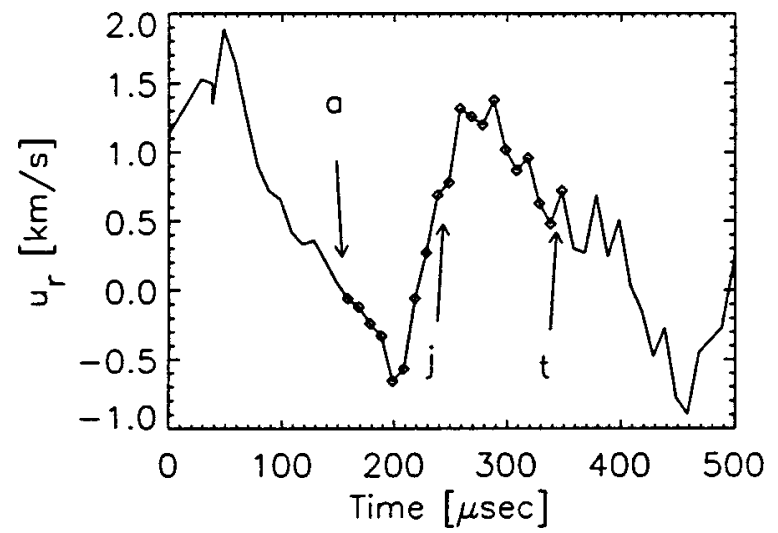

FIG. 15. Plot of measured radial fluid velocity $u_{r}$ near density minimum. The discrete points indicate the sampling times for data shown Fig. 14. $u_{r}$ $<0$ indicates flow from wall to center.

decreasing during the heating cycle, and also indicates that the stochastic heating is fast enough to overcome some cooling due to recycled ions.

We also measured the penetration of cold ions into the torus interior by monitoring the ratio $n_{\text {cold }} / n_{\text {hot }}$ over a range of distances from the chamber wall. At each position, the time evolution of $f(\mathbf{v})$ was sampled in $40 \mu$ s intervals, and the peak cold ion density tabulated for each position. The relative cold ion populations are compared by position in Fig. 16. The figure shows that appreciable cold ion densities were found only near the plasma edge: The relative population of cold ions decreased by about an order of magnitude with every $3 \mathrm{~cm}$ of retreat from the wall. Such localization would be expected for phenomena involving recycling, because recycled ions which penetrate the plasma core should heat stochastically in transit and arrive finally as hot ions indistinguishable from the hot background plasma. (One expects that cold neutrals desorb from the wall before reentering the plasma and re-ionizing.) However, the ionization mean free path $\ell$ in Encore appears to be much longer than the $4 \mathrm{~cm}$ between the wall and the LIF-diagnosed volume. The mean free path is estimated from $\ell=1 /\left(n_{t} \sigma\right)$, where $n_{t}$ is the density of targets $t$ (electrons, ions, or other neutrals), and $\sigma$ is the cross section for a particular ionization event. The cross sections for Ar ionization by charge-

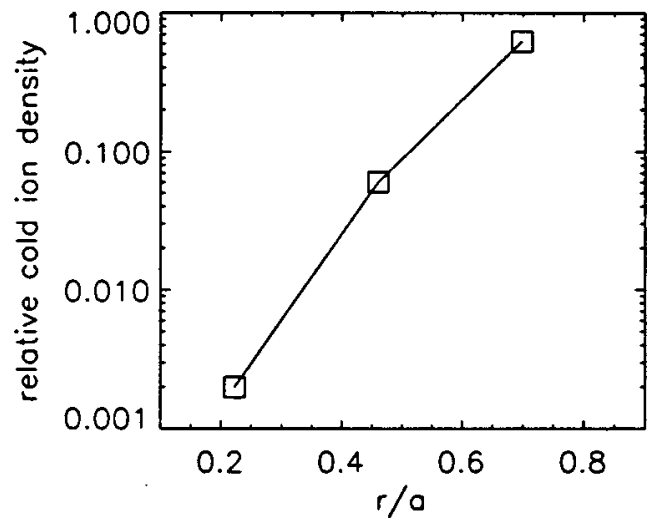

FIG. 16. Dependence of cold ion population on proximity to wall. 
exchange (ce) and by electron impact $(e i)$ are: ${ }^{49} \sigma_{c e} \approx 6$ $\times 10^{-15} \mathrm{~cm}^{2}$ and $\sigma_{e i} \approx 2 \times 10^{-17} \mathrm{~cm}^{2}$. For equal ion and electron densities $n_{e}=n_{i}=10^{12} \mathrm{~cm}^{-3}$, ionization is dominated by charge-exchange, giving $\ell \approx 1.7 \mathrm{~m}$. This length is larger than the torus dimensions, implying that neutrals pervade the vacuum chamber (albeit at low densities) and raising the possibility that recycled particles are ionized in situ, rather than ionized at the wall before convecting inward. In situ ionization, however, would be completely inconsistent with the observed space dependence of the cold particle density (cf. Fig. 16). This discrepancy is unresolved at present. Direct ionization of adsorbed neutrals in collisional desorption events may be the answer, since the density of walladsorbed neutrals is quite high in typical plasma devices. ${ }^{38}$ It is also possible that other ionization channels, e.g., photoionization, can explain the observed high density of cold ions. Further attempts were made to clarify the recycling mechanism by directly following the motion of recycled particles via optical tagging techniques $;^{50}$ however, ion metastable lifetimes were too short to observe significant particle transport.

\section{DISCUSSION}

From the measurements presented in the preceding sections, a complete picture of plasma ion dynamics due to large-amplitude drift waves may be formed. Ions are rapidly and stochastically heated in the plane normal to $\mathbf{B}$, and collisions redistribute the 'stochastic energy' among the three degrees of ion freedom. The anisotropic ion temperature relaxes in accordance with Fokker-Planck theory, with a highly time-dependent collision rate due to the fluctuating plasma density and temperature. Stochastically heated ions are then lost from the plasma when their orbit radii become comparable to the machine dimensions. Such orbit loss results in bulk cooling and density loss. Finally, recycled ions convect inward from the plasma edge and repopulate the depleted plasma.

It is worthwhile to comment further on two aspects of this periodic heating cycle. First, the strongly cyclic nature of the heating seen in Figs. 6 and 7 may at first suggest that ions reversibly exchange energy with the drift wave, provoking questions of whether stochastic heating is true irreversible heating, or whether the heating agent in Encore plasmas is indeed stochasticity. This question is definitively answered by the observation that ion heating is periodic both normal to and along the toroidal field. Recall that along $\mathbf{B}$, the heating agent was found to be not the wave but rather collisions, indisputably an irreversible heating mechanism. Thus the bulk ion cooling cannot be due to reversible energy exchange with the wave and must be due to an external loss mechanism. This picture is consistent with the orbit loss and recycling mechanism described above.

Secondly, our results imply that the ion loss processes essential in the heating-cooling cycle observed here are not unique to the Encore device, but rather should be important in many stochastically heated plasmas. To demonstrate this point, we consider the maximum ion velocities attainable in stochastically heated plasmas, given by Eq. (4). We empha- size that $v_{\max }$ is the half-width of the stochastic region of velocity space centered at $v=0$ (cf. Fig. 5), and therefore this high velocity is accessible to ions in the bulk of the distribution. As noted in Sec. IV C, ions are well-confined by the magnetic field only if their separation $d$ from the wall satisfies $d z v / \Omega$, where $\Omega$ is the (toroidal) ion cyclotron frequency. This is the confinement condition for perfect gyromotion; confinement is expected to be worse for tokamak drift trajectories ${ }^{51}$ and for stochastic ion dynamics, wherein the guiding center approximation breaks down completely. ${ }^{6}$ Inserting $v \approx v_{\max }$ into this expression yields $d \gtrsim(\alpha$ $+1.9) / k_{\perp}$. Finally, noting that $k_{\perp}=m / a$ where $m$ is the wave mode number and $a$ is the machine minor radius, yields the confinement criterion

$$
\frac{d}{a} \geq \frac{\alpha+1.9}{m} \text {. }
$$

Hence for an $m=2$ mode, the confinement condition for stochastically heated ions is $d / a \geqslant \alpha / 2+0.95$, implying that ions must be located more than one machine radius away from the wall to be reasonably well confined at the onset of stochasticity $(\alpha \approx 1)$. In practice, such poor particle confinement may be mitigated by weaker wave fields in the plasma interior (away from the drift wave spatial peak) and other self-consistent wave or chaos-limiting effects. ${ }^{24}$ Nevertheless, Eq. (12) cannot be satisfied by any mode with $m \leqslant 4$, implying that edge effects and ion orbit loss would be expected in any plasma with low mode-number waves and stochastic dynamics. Thus, in light of the observed cyclic nature of the ion heating, cooling, and recycling, it may be speculated that the process heat/loss/cool/recycle is a selfconsistent limit-cycle for the temperature and density fluctuations induced by large-amplitude drift waves. Self limiting mechanisms for particle chaos have been proposed before $^{24}$ but not specifically identified.

\section{ACKNOWLEDGMENTS}

We thank Mr. F. Cosso for invaluable technical assistance. This work was supported by National Science Foundation Grant No. PHY-9413046.

${ }^{1}$ G. R. Smith and A. N. Kaufman, Phys. Rev. Lett. 34, 1613 (1975).

${ }^{2}$ C. F. F. Karney and A. Bers, Phys. Rev. Lett. 39, 550 (1977).

${ }^{3}$ J. F. Drake and T. T. Lee, Phys. Fluids 24, 1115 (1981).

${ }^{4}$ F. Doveil, Phys. Rev. Lett. 46, 532 (1981).

${ }^{5}$ F. Skiff, F. Anderegg, and M. Q. Tran, Phys. Rev. Lett. 58, 1430 (1987).

${ }^{6}$ J. M. McChesney, R. A. Stern, and P. M. Bellan, Phys. Rev. Lett. 59, 1436 (1987).

${ }^{7}$ F. Skiff, F. Anderegg, T. N. Good, P. J. Paris, M. Q. Tran, N. Rynn, and R. A. Stern, Phys. Rev. Lett. 61, 2034 (1988).

${ }^{8}$ A. Fasoli, F. Skiff, R. Kleiber, M. Q. Tran, and P. J. Paris, Phys. Rev. Lett. 70, 303 (1993).

${ }^{9}$ A. D. Bailey III, R. A. Stern, and P. M. Bellan, Phys. Rev. Lett. 71, 3123 (1993).

${ }^{10}$ P.-K. Chia, L. Schmitz, and R. W. Conn, Phys. Plasmas 3, 1569 (1996).

${ }^{11}$ M. Ono, T. Watari, R. Ando, J. Fujita, Y. Hirokura, K. Ida, E. Kako, K. Kawahata, Y. Kawasumi, K. Matsuoka, A. Nishizawa, N. Noda, I. Ogawa, K. Ohkubo, M. Okamato, K. Sato, S. Tanahashi, Y. Taniguchi, T. Tetsuka, K. Toi, and K. Yamazaki, Phys. Rev. Lett. 54, 2339 (1985).

${ }^{12}$ See, for example, H. Weisen, Nucl. Fusion 31, 2247 (1991).

${ }^{13}$ R. A. Stern and J. A. Johnson III, Phys. Rev. Lett. 34, 1548 (1975). 
${ }^{14}$ I. H. Hutchinson, Principles of Plasma Diagnostics (Cambridge University Press, New York, 1987), p. 66.

${ }^{15}$ E. D. Fredrickson and P. M. Bellan, Phys. Fluids 28, 1866 (1985).

${ }^{16}$ S. J. Sanders, P. M. Bellan, and R. A. Stern, Rev. Sci. Instrum. (to be published).

${ }^{17}$ D. G. Swanson, Plasma Waves (Academic Press, New York, 1989), p. 275

${ }^{18}$ G. Schmidt, Physics of High Temperature Plasmas (Academic, New York, 1979), p. 354.

${ }^{19}$ B. B. Kadomtsev, Plasma Turbulence (Academic, London, 1965), pp. 79-80.

${ }^{20}$ J. M. McChesney, P. M. Bellan, and R. A. Stern, Phys. Fluids B 3, 3370 (1991).

${ }^{21}$ A. B. Mikhailovskii, Theory of Plasma Instabilities, Vol. 2 (Consultants Bureau, New York, 1974), p. 52.

${ }^{22}$ This value of the additive term reported here, 1.9 , is between the value 1.5 found in the work by Drake and Lee and the value 2.3 reported by McChesney, Bellan, and Stern.

${ }^{23}$ P. Deeskow, K. Elsasser, and F. Jestczemski, Phys. Fluids B 2, 1551 (1990).

${ }^{24}$ M. Fivaz, A. Fasoli, K. Appert, F. Skiff, M. T. Tran, and M. Q. Tran, Phys. Lett. A 182, 426 (1993).

${ }^{25}$ J. D. Huba, NRL Plasma Formulary (Naval Research Laboratory, Washington DC, 1994), p. 34.

${ }^{26}$ M. N. Rosenbluth, W. M. MacDonald, and D. L. Judd, Phys. Rev. 107, 1 (1957).

${ }^{27}$ N. Rostoker and M. N. Rosenbluth, Phys. Fluids 3, 1 (1960).

${ }^{28}$ V. I. Kogan, in Plasma Physics and the Problem of Controlled Thermonuclear Reactions Vol. 1, edited by M. A. Leontovich (Pergamon, New York, 1961), p. 157.

${ }^{29}$ L. Spitzer, Jr., Physics of Fully Ionized Gases (Wiley, New York, 1962), pp. $132-133$.

${ }^{30}$ A. W. Hyatt, C. F. Driscoll, and J. H. Malmberg, Phys. Rev. Lett. 59, 2975 (1987).

${ }^{31}$ B. R. Beck, J. Fajans, and J. H. Malmberg, Phys. Rev. Lett. 68, 317 (1992).

${ }^{32}$ F. Anderegg et al., Phys. Rev. Lett. 78, 2128 (1997).

${ }^{33}$ The relatively poor agreement between theory and experiment in the first heating cycle $(t \leqslant 100 \mu \mathrm{s})$ probably results from overestimation of ion density by the Langmuir probe: Analysis of the probe data using the method of Laframboise [see National Technical Information Service document No. AD 634596 (J. G. Laframboise, "Theory of spherical and cylindrical Langmuir probes in a collisionless Maxwellian plasma at rest,"
University of Toronto Institute for Aerospace Studies Report No. 100, 1966). Copies may be obtained from the National Technical Information Service, Springfield, VA 22161] indicates that $n(t \leqslant 100 \mu \mathrm{s})$ is overestimated by about $20 \%$ relative to $n(t \gtrsim 100 \mu \mathrm{s})$.

${ }^{34}$ B. Unterberg, H. Knauf, P. Bogen, E. Hintz, A. Pospieszczyk, D. Reiter, D. Rusbuldt, and U. Samm, J. Nucl. Mater. 220-222, 462 (1995).

${ }^{35}$ J. N. Brooks, Phys. Plasmas 3, 2286 (1996).

${ }^{36}$ J. A. Wesson, Tokamaks (Oxford University Press, Oxford, 1987), p. 75.

${ }^{37}$ The distribution in Fig. 13(a) provokes the question of what temperature means in the context of non-Maxwellian distributions. This question is answered qualitatively by noting that the distribution in Fig. 13(a) is certainly hotter than that in Fig. 3(c), in the sense that it is much broader.

${ }^{38}$ G. M. McCracken and P. E. Stott, Nucl. Fusion 19, 889 (1979); P. C. Stangeby and G. M. McCracken, ibid. 30, 1271 (1990).

${ }^{39}$ Q. M. Zhang and J. Z. Larese, Phys. Rev. B 43, 938 (1991).

${ }^{40}$ G. Dujardin, L. Hellner, L. Philippe, M. J. Besnardramage, and P. Cirkel, Phys. Rev. B 48, 14529 (1993).

${ }^{41}$ M. Headgordon, J. C. Tully, H. Schlichting, and D. Menzel, J. Chem. Phys. 95, 9266 (1991)

${ }^{42}$ W. Friess, T. Brunner, and D. Menzel, Surf. Sci. 309, 182 (1994).

${ }^{43}$ W. M. Lau, I. Bello, L. J. Huang, X. Feng, M. Vos, and I. V. Mitchell, J. Appl. Phys. 74, 7101 (1993).

${ }^{44}$ K. Erents and G. Carter, Vacuum 17, 215 (1967).

${ }^{45}$ K. Erents and G. Carter, Br. J. Appl. Phys., J. Phys. D 2, 435 (1969).

${ }^{46} \mathrm{We}$ should note that the measured radial dependence of electron temperature $T_{e}$ in Encore is fairly uniform over the range $r / a=0.13$ to $r / a$ $=0.83$. Because the populations of argon metastables are determined primarily by $T_{e}$, this uniformity implies that the fractional population of the $3 d^{2} G_{9 / 2}$ state used for LIF should be reasonably consistent among the recycled and background plasma particles.

${ }^{47}$ B. A. Trubnikov, in Reviews of Plasma Physics Vol. 1, edited by M. A. Leontovich (Consultants Bureau, New York, 1965), pp. 186 and 190.

${ }^{48}$ G. Schmidt, Physics of High Temperature Plasmas (Academic, New York, 1979), p. 387.

${ }^{49}$ S. C. Brown, Basic Data of Plasma Physics, 2nd ed. (M.I.T., Cambridge, Massachusetts, 1967), pp. 73 and 143.

${ }^{50}$ R. A. Stern, D. N. Hill, and N. Rynn, Phys. Lett. A 93, 127 (1983).

${ }^{51}$ Here we have treated particle confinement as being determined by the toroidal component of the magnetic field $B$. For $T_{\|} \approx T_{\perp}$ and $B_{\text {poloidal }}$ $<B_{\text {toroidal }}$ this assumption overestimates particle confinement. Hence the orbit-loss criterion Eq. (12) is conservative. 